\title{
On the Solutions of Schlömilch's Integral Equations
}

\author{
Ahmet Altürk \\ Amasya University, Faculty of Arts and Sciences, Department of Mathematics, İpekköy, \\ Amasya, +90 2421613, \\ ahmet.alturk@amasya.edu.tr \\ Recieved: 12 January 2017 \\ Accepted: 15 June 2017 \\ DOI: $10.18466 /$ cbayarfbe.339329
}

\begin{abstract}
The linear Schlömilch's integral equation is an important and useful equation in atmospheric and terrestrial physics. The equation and its solution have been used for some ionospheric problems. It can also be considered as a special type of Fredholm integral equation of the first kind. This correspondence allows one to use the mathematical tools available for solving Fredholm integral equation of the first kind. In this article, we provide an alternative closed-form expression for solutions of the linear and the nonlinear Schlömilch's integral equation in terms of the well-known gamma function. Some elaborate examples are provided to demonstrate the simplicity and applicability of the proposed formulae.
\end{abstract}

Keywords - Schlömilch's Integral Equation, Fredholm Integral Equation of the First Kind, Terrestrial Physics, Gamma Function.

\section{Introduction}

Many physical processes can be expressed mathematically as a Fredholm integral equation of the first kind. The Schlömilch's integral equation is one example. It is an important and useful equation in atmospheric and terrestrial physics. For the derivation and applications of the equation in physics, we refer the reader to [1-7].

The standard linear Schlömilch's integral equation has an unknown function with a complicated argument and admits the following form:

$$
f(x)=\frac{2}{\pi} \int_{0}^{\pi / 2} \phi(x \sin \theta) d \theta, \quad-\pi \leq x \leq \pi .
$$

It's been shown that this equation provides a solution which has the following form:

$$
\phi(x)=f(0)+x \int_{0}^{\pi / 2} f_{\epsilon}^{\prime}(x \sin \theta) d \theta, \quad \epsilon=x \sin \theta,
$$

where the derivative is taken with respect to $\epsilon=x \sin \theta[1$ 4].

There are more methods to deal with Fredholm integral equation of the second kind than the first kind. Therefore, when seeking for a solution of a first kind integral equation, it is almost a standard approach to try to convert it into a second kind. This is usually done by using some regularization or iterative methods. In [6], the author used the regularization method introduced independently by Tikhonov
[8,9] and Phillips [10] to transform the Schlömilch's integral equation to a second kind integral equation, and then applied the Adomian decomposition method [11,12] to obtain the solution. In [4], the authors introduced a new iterative procedure in order for converting the integral equation to a form that is suitable for applying the Adomian decomposition method. In [5], the authors proposed a new method based on the fractional order of the Chebyshev functions. However, all approaches require a great deal of calculation even for some uncomplicated data function $\boldsymbol{f}$.

We aim to obtain a simple formula which provides the solution for Schlömilch's integral equation when the data function $\boldsymbol{f}$ has a special form. We also investigate the relation between the gamma function and the solution of the Schlömilch's integral equation. The results in this article include closed-form expression for solution of standard, generalized, and nonlinear Schlömilch's integral equation. In addition, numerical examples are provided to show the ability and simplicity of the method.

The organization of the article is as follows:

In section 2, we provide some basic concepts which will be needed for the subsequent sections. In section 3, we introduce a method of solution for

- the linear Schlömilch's integral equation,

- the generalized Schlömilch's integral equation, and

- the nonlinear Schlömilch's integral equation. 
The final section presents a conclusion for this work and a direction for future studies.

\section{Review of Basic Concepts}

Before delving into the details of the proposed method, we want to set up some preliminary consepts which will show up throughout the article.

\section{Definition 1.}

$$
\Gamma(t)=\int_{0}^{\infty} x^{t-1} e^{-x} d x, \quad t \in(0, \infty),
$$

when this integral converges [13].

Theorem 1. [13]

If $x, y \in(0, \infty)$, then

$$
\int_{0}^{\pi / 2} \cos ^{2 x-1} \theta \sin ^{2 y-1} \theta d \theta=\frac{\Gamma(x) \Gamma(y)}{2 \Gamma(x+y)}
$$

Theorem 2. [13]

For each $x \in(0, \infty)$,

- $\quad \boldsymbol{\Gamma}(\boldsymbol{x})$ exists and is finite,

- $\quad \Gamma(x+1)=x \Gamma(x)$, and

- for $n \in \square, \Gamma(n)=(n-1)$ !

\section{Theorem 3. (Wallis' Product [15])}

$$
\frac{2}{\pi}=\frac{1 \cdot 3}{2 \cdot 2} \cdot \frac{3 \cdot 5}{4 \cdot 4} \cdot \frac{5 \cdot 7}{6 \cdot 6} \cdot \ldots \cdot \frac{(2 n-1) \cdot(2 n+1)}{2 n \cdot 2 n} \cdot \ldots .
$$

The gamma function crops up in many areas of physics, engineering and applied mathematics. It plays a significant role in the theory of differential equations. It is used both in finding and expression of solutions of many differential equations. Not surprisingly, it plays an important role in the theory of integral equations as well. One of the aim of this article is to establish the connection between the solution of Schlömilch's integral equation and the gamma function.

\section{Method of Solution}

In what follows, we investigate standard, generalized, and nonlinear Schlömilch's integral equation, respectively.

\subsection{The Linear Schlömilch's integral equation}

We consider the following Schlömilch's integral equation:

$$
f(x)=\frac{2}{\pi} \int_{0}^{\pi / 2} \phi(x \sin \theta) d \theta, \quad-\pi \leq x \leq \pi .
$$

Let us start with two simple cases that $\boldsymbol{f}(\boldsymbol{x})$ can take. Observe that as $\boldsymbol{f}(\boldsymbol{X})$ has a constant term, we can remove it by assuming the unknown function $\phi$ has the same constant term, and then solving an equivalent equation. This simply helps reducing an amount of calculation in solving the equation.

Lemma 1.

If $\boldsymbol{f}(\boldsymbol{x})$ has a constant term, i.e; $f(x)=g(x)+\boldsymbol{c}$, then $\phi(x)=\psi(x)+C$ is a solution of (1.1),

where $\boldsymbol{\psi}(\boldsymbol{X})$ is a solution of

$$
g(x)=\frac{2}{\pi} \int_{0}^{\pi / 2} \psi(x \sin \theta) d \theta, \quad-\pi \leq x \leq \pi .
$$

Before considering the general case, we now consider the following special case.

\section{Lemma 2.}

If $f(x)=a_{0}+a_{1} x+g(x)$, then

$\phi(x)=a_{0}+\frac{\pi a_{1}}{2} x+\psi(x)$ is a solution of (1.1),

where $\boldsymbol{\psi}(\boldsymbol{x})$ is a solution of

$$
g(x)=\frac{2}{\pi} \int_{0}^{\pi / 2} \psi(x \sin \theta) d \theta, \quad-\pi \leq x \leq \pi
$$

Let $\boldsymbol{f}$ be a polynomial function of degree $\boldsymbol{N}$, i.e.,

$$
\begin{aligned}
f(x) & =\sum_{k=0}^{N} a_{k} x^{k} \text { and let } \\
d_{0} & =a_{0}, \quad d_{1}=\frac{\pi a_{1}}{2}, d_{k}=c_{k} \times a_{k}, k \geq 2,
\end{aligned}
$$

where

$c_{k}= \begin{cases}\frac{k}{k-1} \times \frac{k-2}{k-3} \times \cdots \times \frac{2}{1}, & \mathrm{k} \text { even, } \\ \frac{k}{k-1} \times \frac{k-2}{k-3} \times \cdots \times \frac{3}{2} \times \frac{\pi}{2}, & \mathrm{k} \text { odd } .\end{cases}$

Define $\phi(x)=\sum_{k=0}^{N} d_{k} x^{k}$. It can easily be verified that $\phi$ is a solution of (1.1). From the lemma 1 and 2, we know that $d_{0}=a_{0}$ and $d_{1}=\frac{\pi a_{1}}{2}$.

For $k \geq 2$, the problem reduces to computing

$$
\int_{0}^{\pi / 2} \sin ^{k}(\theta) d \theta
$$

Evaluating this integral and equating like terms we get the desired coefficients. Since the solution is unique [6], we can conclude that when $\boldsymbol{f}$ is a polynomial function of degree $N, \phi$ must be a polynomial function of the same degree. 
Conversely, we assume that $\phi$ is a polynomial function of degree $\boldsymbol{N}$. Substitute $\phi$ into (1.1), we obtain a polynomial function of degree $\boldsymbol{N}$. Thus, $\boldsymbol{f}$ must be a polynomial function of the same degree. Therefore, we have the following theorem.

\section{Theorem 4.}

$\boldsymbol{f}$ is a polynomial function of degree $\boldsymbol{N}$ if and only if the solution of (1.1) is a polynomial function of the same degree.

It is important to notice that (1.5) has a special value. The reader may have also noticed that it is this integral that was used by John Wallis in obtaining his celebrated product formula for $2 / \pi$. For more information on this, we refer the interested redears to $[14,15]$ and the references therein. Furthermore, it is related to the gamma function. To be more precise, the coefficients $\boldsymbol{c}_{\boldsymbol{k}}$ can also be obtained from the following formula:

$$
c_{k}=\frac{\sqrt{\pi} \Gamma(k / 2+1)}{\Gamma\left(\frac{k+1}{2}\right)}, k=0,1, \ldots, N .
$$

This formula is just a simple consequence of relation between the gamma function and the integral of powers of the sine function. So, when $\boldsymbol{f}$ is a polynomial function, the solution is readily available and it can be expressed in terms of the gamma function.

\section{Example 1.}

Consider the following Schlömilch's integral equation [4]:

$$
1+x=\frac{2}{\pi} \int_{0}^{\pi / 2} \phi(x \sin \theta) d \theta, \quad-\pi \leq x \leq \pi .
$$

The equation (1.7) is of the form of (1.1). Since $\mathbf{a}_{\mathbf{0}}=\mathbf{1}$ and $\mathbf{a}_{\mathbf{1}}=\mathbf{1}$, from the theorem 4 , we directly obtain the solution. That is,

$$
d_{0}=a_{0}=1 \text { and } d_{1}=\frac{\pi}{2} a_{1}=\pi / 2 .
$$

Alternatively, one can use (1.6) to obtain

$d_{0}=\frac{\sqrt{\pi} \Gamma(1)}{\Gamma(1 / 2)} a_{0}=1$ and $d_{1}=\frac{\sqrt{\pi} \Gamma(3 / 2)}{\Gamma(1)} a_{1}=\pi / 2$

It can easily be verified that

$$
\phi(x)=d_{0}+d_{1} x=1+\frac{\pi}{2} x
$$

is a solution of (1.7).

\section{Example 2.}

Consider the following Schlömilch's integral equation [6]:

$$
1+\pi x^{2}=\frac{2}{\pi} \int_{0}^{\pi / 2} \phi(x \sin \theta) d \theta, \quad-\pi \leq x \leq \pi \text {. (1.8) }
$$

The equation (1.8) is of the form of (1.1). Since $\mathbf{a}_{0}=\mathbf{1}, \mathbf{a}_{1}=\mathbf{0}$, and $\mathbf{a}_{2}=\boldsymbol{\pi}$, from the theorem 4 , we directly obtain the solution, namely,

$$
d_{0}=a_{0}=1, d_{1}=\frac{\pi}{2} a_{1}=0 \text {, and } d_{2}=2 a_{2}=2 \pi .
$$

Alternatively, one can use (3.6) to obtain

$$
\begin{aligned}
& d_{0}=\frac{\sqrt{\pi} \Gamma(1)}{\Gamma(1 / 2)} a_{0}=1, \\
& d_{1}=\frac{\sqrt{\pi} \Gamma(3 / 2)}{\Gamma(1)} a_{1}=0, \\
& d_{2}=\frac{\sqrt{\pi} \Gamma(2)}{\Gamma(3 / 2)} a_{2}=2 \pi .
\end{aligned}
$$

It is easy to verify that

$$
\phi(x)=d_{0}+d_{1} x+d_{2} x^{2}=1+2 \pi x^{2}
$$

is a solution of (3.8).

We aim to extend the foregoing idea further and obtain a similar formula for other types of Schlömilch's integral equation. For instance, in some cases, the Schlömilch's integral equation can also appear as

$$
f(x)=\frac{2}{\pi} \int_{0}^{\pi / 2} \phi(x \sin (m \theta)) d \theta, \quad-\pi \leq x \leq \pi
$$

$$
\text { and } m=2,3, \ldots \text {. }
$$

To handle this type of equations and express their solutions as a simple formula, we use a simple change of variables. That is, letting $\boldsymbol{m} \boldsymbol{\theta}=\boldsymbol{t}$ transforms (1.9) to

$$
f(x)=\frac{2}{m \pi} \int_{0}^{m \pi / 2} \phi(x \sin t) d t, \quad-\pi \leq x \leq \pi . \text { (1.10) }
$$

Notice that upper limit of integration is now $m \pi / 2$. This will introduce a new term in the formula.

\section{Theorem 5.}

If $\boldsymbol{f}(\boldsymbol{x})$ is a polynomial function, i.e;

$$
f(x)=\sum_{k=0}^{N} a_{k} x^{k} \text { and } a=\int_{0}^{m \pi / 2} \sin (t) d t \neq 0
$$

then $\phi(x)=\sum_{k=0}^{N} d_{k} x^{k}$ is a solution of (1.9), where 
$d_{0}=a_{0}, d_{1}=\frac{m \pi}{2 a} a_{1}$, and for $k \geq 2$

$c_{k}= \begin{cases}\frac{k}{k-1} \times \frac{k-2}{k-3} \times \ldots \times \frac{2}{1}, & \mathrm{k} \text { is even, } \\ \frac{k}{k-1} \times \frac{k-2}{k-3} \times \ldots \times \frac{3}{2} \times \frac{m \pi}{2 a}, & \mathrm{k} \text { is odd, }\end{cases}$

and $d_{k}=c_{k} \times a_{k}$.

Proof: The proof is similar to that of theorem 4 .

A few remarks are in order.

\section{Remark 1.}

The case where $\mathbf{a}=\mathbf{0}\left(o r, m=4 n, n \in \square^{+}\right)$implies that the solution cannot have odd power of monomials.

\section{Remark 2.}

If the data function $\boldsymbol{f}$ involves components of odd power and $\mathbf{a}=\mathbf{0}$, then the solution of the form

cannot be achieved.

$$
\phi(x)=\sum_{k=0}^{N} d_{k} x^{k}
$$

We want to note that the above coefficients can be expressed in terms of the gamma function as follows:

$$
c_{k}= \begin{cases}\frac{\sqrt{\pi} \Gamma(k / 2+1)}{\Gamma\left(\begin{array}{c}
k+1 \\
2
\end{array}\right)}, & k \text { is even, } \\
\frac{m \sqrt{\pi} \Gamma(k / 2+1)}{a \Gamma\left(\frac{k+1}{2}\right)}, & k \text { is odd. }\end{cases}
$$

\section{Example 3.}

Consider the following Schlömilch's integral equation [6]:

$$
2 x=\frac{2}{\pi} \int_{0}^{\pi / 2} \phi(x \sin 3 \theta) d \theta, \quad-\pi \leq x \leq \pi .
$$

The equation (1.12) is of the form of (1.9) with $\boldsymbol{m}=\mathbf{3}$. Since $\boldsymbol{a}_{\mathbf{0}}=\mathbf{0}, \boldsymbol{a}_{\mathbf{1}}=\mathbf{2}$, and $\boldsymbol{a}=\mathbf{1}$, from the theorem 5 , we directly obtain the solution. That is,

$$
d_{0}=a_{0}=0 \text { and } d_{1}=\frac{m \pi}{2 a} a_{1}=3 \pi .
$$

Alternatively, one can use (1.11) to obtain

$d_{0}=\frac{\sqrt{\pi} \Gamma(1)}{\Gamma(1 / 2)} a_{0}=0$ and $d_{1}=\frac{m \sqrt{\pi} \Gamma(3 / 2)}{a \Gamma(1)} a_{1}=3 \pi$.

It is easy to verify that

$$
\phi(x)=d_{0}+d_{1} x=3 \pi x
$$

is a solution of (1.12).

\section{Example 4.}

Consider the following Schlömilch's integral equation [4]:

$$
1+x^{2}=\frac{2}{\pi} \int_{0}^{\pi / 6} \phi(x \sin 3 \theta) d \theta, \quad-\pi \leq x \leq \pi .
$$

Letting $t=3 \theta$, (1.13) becomes

$$
3+3 x^{2}=\frac{2}{\pi} \int_{0}^{\pi / 2} \phi(x \sin t) d t, \quad-\pi \leq x \leq \pi,
$$

which is of the form of (1.1). Since $\mathbf{a}_{0}=3, \mathbf{a}_{1}=\mathbf{0}$, and $a_{2}=3$, we have

$$
d_{0}=a_{0}=3, d_{1}=\frac{\pi}{2} a_{1}=0, \text { and } d_{2}=2 a_{2}=6 .
$$

It is easy to verify that

$$
\phi(x)=d_{0}+d_{1} x+d_{2} x^{2}=3+6 x^{2}
$$

is a solution of (1.13).

\subsection{The Generalized Schlömilch's integral equation}

The generalized Schlömilch's integral equation has the following form:

$$
f(x)=\frac{2}{\pi} \int_{0}^{\pi / 2} \phi\left(x \sin ^{n} \theta\right) d \theta, \quad n \geq 1 .
$$

We are investigating the solution of the form

$$
\phi(x)=\sum_{m=0}^{N} d_{m} x^{m} \text { for } n=1,2, \ldots
$$

\section{Theorem 6.}

If $\boldsymbol{f}$ is a polynomial function, i.e;

$$
f(x)=\sum_{k=0}^{N} a_{k} x^{k} \text {, then } \phi(x)=\sum_{m=0}^{N} d_{m} x^{m}
$$

is a solution of (1.14), where $d_{0}=a_{0}, d_{m}=c_{n \times m} \times a_{m}$ 
for $m \geq 1, c_{n \times m}$ is the same as defined in (1.4).

Proof: The proof is similar to that of theorem 4.

\section{Example 5.}

Consider the following Schlömilch's integral equation [6]: $x+3 x^{2}=\frac{2}{\pi} \int_{0}^{\pi / 2} \phi\left(x \sin ^{2} \theta\right) d \theta, \quad-\pi \leq x \leq \pi$. (1.15)

The equation (1.15) is of the form of (3.14) with $\boldsymbol{n}=2$. Since $\boldsymbol{a}_{\mathbf{0}}=\mathbf{0}, \mathbf{a}_{\mathbf{1}}=\mathbf{1}$, and $\boldsymbol{a}_{\mathbf{2}}=\mathbf{3}$, from the theorem 6 , we directly obtain the solution. That is,

$$
d_{0}=a_{0}=0, d_{1}=c_{2} \times a_{1}=2, \text { and } d_{2}=c_{4} \times a_{2}=8 \text {. }
$$

Alternatively, one can use (1.6) to obtain

$$
\begin{aligned}
& c_{0}=\frac{\sqrt{\pi} \Gamma(1)}{\Gamma(1 / 2)}=1, \\
& c_{2}=\frac{\sqrt{\pi \Gamma(3 / 2)}}{\Gamma(1)}=2, \\
& c_{4}=\frac{\sqrt{\pi} \Gamma(3)}{\Gamma(5 / 2)}=\frac{8}{3} .
\end{aligned}
$$

By using $d_{m}=c_{m \times m} \times a_{m}$, it is easy to verify that

$$
\phi(x)=d_{0}+d_{1} x+d_{2} x^{2}=2 x+8 x^{2}
$$

is a solution of (1.15).

\section{Example 6.}

Consider the following Schlömilch's integral equation [6]:

$$
4 x-\frac{5 x^{2}}{16}=\frac{2}{\pi} \int_{0}^{\pi / 2} \phi\left(x \sin ^{3} \theta\right) d \theta, \quad-\pi \leq x \leq \pi \text {. }
$$

The equation (1.16) is of the form of (1.14) with $\boldsymbol{n}=\mathbf{3}$. Since $a_{0}=0, a_{1}=4$, and $a_{2}=-5 / 16$, from the theorem 6 , we directly obtain the solution, namely,

$d_{0}=a_{0}=0, d_{1}=c_{3} \times a_{1}=3 \pi$, and $d_{2}=c_{6} \times a_{2}=-1$. Alternatively, one can use (1.6) to obtain

$$
\begin{aligned}
& c_{0}=\frac{\sqrt{\pi} \Gamma(1)}{\Gamma(1 / 2)}=1, \\
& c_{3}=\frac{\sqrt{\pi} \Gamma(5 / 2)}{\Gamma(2)}=\frac{3 \pi}{4}, \\
& c_{6}=\frac{\sqrt{\pi} \Gamma(4)}{\Gamma(7 / 2)}=\frac{16}{5} .
\end{aligned}
$$

By using $d_{m}=c_{m \times m} \times a_{m}$, it is easy to verify that

$$
\phi(x)=d_{0}+d_{1} x+d_{2} x^{2}=3 \pi x-x^{2}
$$

is a solution of (1.16).

\subsection{The Nonlinear Schlömilch's integral equation}

We consider the nonlinear Schlömilch's integral equation which has the following form:

$$
f(x)=\frac{2}{\pi} \int_{0}^{\pi / 2} F(\phi(x \sin \theta)) d \theta, \quad-\pi \leq x \leq \pi,(1.17)
$$
where $F(\phi(x \sin \theta))$ is a nonlinear function of $\phi(x \sin \theta)$.

We assume that $F$ is invertible so that letting that

$$
F(\phi(x \sin \theta))=\psi(x \sin \theta)
$$

will imply that $\phi(x \sin \theta)=F^{-1}(\psi(x \sin \theta))$. Thus, with this transformation, (1.17) becomes

$$
f(x)=\frac{2}{\pi} \int_{0}^{\pi / 2} \psi(x \sin \theta) d \theta
$$

which is equivalent to (1.1). We solve this equation for $\boldsymbol{\psi}(\boldsymbol{x})$ and then use the inverse transform $\boldsymbol{F}^{-1}$ to get $\phi(x)$.

\section{Example 7.}

Consider the following nonlinear Schlömilch's integral equation [6]:

$$
5 x^{6}=\frac{2}{\pi} \int_{0}^{\pi / 2} \phi^{2}(x \sin \theta) d \theta, \quad-\pi \leq x \leq \pi .
$$

Letting that $\psi=\phi^{2}$, the equation (1.18) becomes

$$
5 x^{6}=\frac{2}{\pi} \int_{0}^{\pi / 2} \psi(x \sin \theta) d \theta, \quad-\pi \leq x \leq \pi,
$$

which is of the form of (1.1). Since $\mathbf{a}_{6}=5$ and all the other $\boldsymbol{a}_{\boldsymbol{k}}$ 's are zero, from the theorem 4 , we directly obtain the solution. That is, 


$$
d_{6}=c_{6} \times a_{6}=16
$$

Alternatively, one can use (1.6) to obtain

$$
d_{6}=\frac{\sqrt{\pi} \Gamma(4)}{\Gamma(7 / 2)} a_{6}=16
$$

Therefore, $\psi(x)=16 x^{6}$ is a solution of (1.19). Inverting the transform that $\psi=\phi^{2}$ results in $\phi=\sqrt{\psi}$. It can easily be verified that

$$
\phi(x)=\sqrt{\psi}= \pm 4 x^{3}
$$

is a pair of solution of (1.18).

\section{Example 8.}

Consider the following nonlinear Schlömilch's integral equation [6]:

$$
\frac{35 x^{8}}{8}=\frac{2}{\pi} \int_{0}^{\pi / 2} \phi^{4}(x \sin \theta) d \theta, \quad-\pi \leq x \leq \pi .
$$

Letting that $\psi=\phi^{4}$, the equation (1.20) becomes

$$
\frac{35 x^{8}}{8}=\frac{2}{\pi} \int_{0}^{\pi / 2} \psi(x \sin \theta) d \theta,-\pi \leq x \leq \pi,
$$

which is of the form of (1.1). Since $\mathbf{a}_{\mathbf{8}}=\mathbf{8}$ and all the other $\boldsymbol{a}_{\boldsymbol{k}}$ 's are zero, from the theorem 4 , we directly obtain the solution. That is,

$$
d_{8}=c_{8} \times a_{8}=16
$$

Alternatively, one can use (1.6) to obtain

$$
d_{8}=\frac{\sqrt{\pi} \Gamma(5)}{\Gamma(9 / 2)} a_{8}=16
$$

Therefore, $\psi(x)=16 x^{8}$ is a solution of (1.21). Inver ting the transform that $\psi=\phi^{4}$ results in $\phi=\sqrt{\psi}$. It can easily be verified that

$$
\phi(x)=\sqrt{\psi}= \pm 2 x^{2}
$$

is a pair of solution of (1.20).

\section{Conclusion}

The Schlömilch's integral equation is an important and useful equation in atmospheric and terrestrial physics. In this study, we establish a connection between the solution of

\section{A. Altürk}

various kind of Schlömilch's integral equation and the wellknown gamma function. In other words, we obtain an alternative formula to the solution of Schlömilch's integral equation. In order to compare the results and to show the simplicity of the proposed formulae, some examples are chosen from $[4,6]$. For a future study, we aim to extend the results obtained in this article further and to establish a similar connection for less restrictive data functions.

\section{References}

1. Unz, H, Schlömilch's Integral Equation, Journal of Atmospheric and Terrestrial Physics, 1963, 25, 101-102.

2. Unz, H, Schlömilch's Integral Equation for Oblique Incidence, Journal of Atmospheric and Terrestrial Physics, 1966, 28, 315-316.

3. Gething, P.J.D, Maliphant, R.G, Unz's Application of Schlomilch's Integral Equation to Oblique Incidence Observations, Journal of Atmospheric and Terrestrial Physics, 1967, 29, 599-600.

4. Bougoffa, L, Al-Hagbani, M, Brceski, I, Randolph, C.R.A, Convenient Technique for Solving Integral Equations of the First Kind by the Adomian Decomposition Method, Kybernetes, 2012, 41, 145-156.

5. Parand, K, Delkosh, M, Solving the Nonlinear Schlomilch's Integral Equation Arising in Ionospheric Problems, Afrika Matematika, 2016, doi: 10.1007/s13370-016-0459-3.

6. Wazwaz, A, Solving Schlömilch's Integral Equation by the Regularization-Adomian Method, Romanian Journal of Physics, 2015, 60, 56 - 71.

7. De, S.S, Sarkar, B.K, Manasi, M, De, M, Gosh, B, Adhikari, S.K, On Schlomilch's Integral Equation for the Ionospheric Plasma, Japanese Journal of Applied Physics, 1994, 33, 1-7A.

8. Tikhonov, A.N, Solution of Incorrectly Formulated Problems and the Regularization Method, Soviet Mathematics Doklady, 1963, 4, 1035-1038.

9. Tikhonov, A.N, Regularization of Incorrectly Posed Problems, Soviet Mathematics Doklady, 1963, 4, 1624-1627.

10. Philips, D.L. A, Technique for the Numerical Solution of Certain Integral Equations of the First Kind, Journal of the Association for Computing Machinery, 1962, 84-96.

11. Adomian, G, Solving Frontier Problems of Physics, the Decomposition Method, 1994; Kluwer, Boston.

12. Wazwaz, A, Linear and Nonlinear Integral Equations: Methods and Applications; Springer and Hep: Berlin and Beijing, 2011; pp 658.

13. Wade, W.R, An Introduction to Analysis; Pearson Prentice Hall: New Jersey, 2010; pp 680.

14. Wastlund, J, An Elementary Proof of the Wallis Product Formula for Pi. The mathematical association of America, 2007; 114, 914-917.

15. Khrushchev, S, A Recovery of Brouncker's Proof for the Quadrature Continued Fraction, Publicacions Matematiques, 2006, 50, 3-42. 